\title{
Microdosimetric measurements as a tool to assess potential in- and out-of-field toxicity regions in proton therapy
}

\author{
M Missiaggia ${ }^{1}$, G Cartechini $^{1}$, E Scifoni $^{2}$, M Rovituso ${ }^{3}$, F

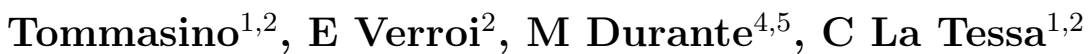 \\ ${ }^{1}$ University of Trento, Via Sommarive 14, 38123 Trento, Italy \\ ${ }^{2}$ Trento Institute of Fundamental Physics and Applications (TIFPA), Via \\ Sommarive 14, 38123 Trento, Italy \\ ${ }^{3}$ HollandPTC Proton Therapy Center, Delft, Netherlands \\ ${ }^{4}$ GSI Helmholtzzentrum für Schwerionenforschung, Planckstrasse 1, 64291 \\ Darmstadt, Germany \\ ${ }^{5}$ Technische Universität Darmstadt, Darmstadt, Germany \\ E-mail: chiara.latessa@unitn.it
}

\begin{abstract}
Variations of the Relative Biological Effectiveness (RBE) are allegedly one of the primary causes of unexpected normal tissue toxicities during tumor treatments with charged particles. Unlike carbon therapy, where the treatment planning are optimized on the bases of the RBE-weighted dose, a constant RBE value of 1.1 is currently used in proton therapy. Assuming a uniform value can lead to under- or over-dosage, not just to the tumor but also to the surrounding normal tissue. In this study, we take advantage of an existing methodology for assessing cell survival RBE from measured physical quantities and exploited it to assess potential toxicity regions both inside and outside the irradiation field. We used microdosimetry to measure lineal energy $y$ spectra in a water phantom irradiated with a $152 \mathrm{MeV}$ clinical proton beam. This approach provided a simultaneous characterization of the radiation field quality as well as an estimate of the deposited dose. Taking advantage of the Linear Quadratic (LQ) and a modified version of the Microdosimetric Kinetic (MKM) models, the microdosimetric data were combined with radiobiological parameters ( $\alpha$ and $\beta$ ) characteristics of Human Salivary Gland (HSG) tumor cells for assessing cell survival RBE and RBE-weighted dose at several depths in-beam and at out-of-field. For a full treatment of 60 Gy delivered to the tumor, the overall dose received by the normal tissue is as high as 4 Gy at the field edge, $10^{-2} \mathrm{~Gy}$ in the close-out-of-field region and $5^{*} 10^{-4}$ Gy in the far-outof-field region. The RBE measured in-beam shows large variations, ranging from
\end{abstract}


$0.98 \pm 0.18$ at the plateau to $2.68 \pm 0.10$ at the tail. Out-of-field, the values are always higher than 1.1 independently of the depth and reach their maximum value of approximately 2.6 at the Bragg peak depth. The approach presented in this study provides a characterization of the radiation field in-beam and out-of-field from which the RBE for cell survival can be calculated. The results can point to normal tissue regions at potential higher risk of toxicities.

Submitted to: Physics in Medicine and Biology

Keywords: microdosimetry, proton therapy, out-of-field dose, RBE, toxicity 


\section{Introduction}

Proton therapy is a widespread and yet still emerging technology in cancer treatment. Its main advantage relies on the proton physical properties, which translate into a higher ability to spare normal tissue compared to conventional radiotherapy. In addition, protons have a slightly enhanced radiobiological effectiveness, especially in cell killing ((Tommasino \& Durante 2015)). This feature is described by the Relative Biological Effectiveness (RBE) parameter, defined as the dose ratio between a reference radiation (generally $\mathrm{x}$-rays) and the test radiation to induce the same biological effect. In vivo and in vitro data have indicated that therapeutic protons have an average RBE of 1.1, which is currently used clinically (Paganetti 2014). Assuming a uniform value independent of the beam physical parameters, such as Linear Energy Transfer (LET) or dose, however, can lead to under- or over-dosage, not just of the tumor but also of the surrounding normal tissue and can potentially impact the clinical effectiveness of proton therapy. Overdosages are acceptable in the tumor but they can cause severe toxicity in the healthy tissue. The biological uncertainty becomes even more critical in hypofractionation regiments and thus represents one of the limits for dose escalation (Friedrich et al. 2014). Following the same approach used in carbon therapy ((Durante \& Loeffler 2010)), RBE variations can be taken into account by optimizing the treatment planning in terms of RBEweighted dose (i.e. RBE multiplied by the physical dose). For this reason, assessing the RBE and its uncertainty has been emerging as an important topic and has required experimental and theoretical effort for its full characterization.

Establishing a relationship between RBE and physical parameters such as physical dose or LET, which can be measured directly, is the most promising approach in the field ((Paganetti 2014)). A collection of data extracted from the particle irradiation database ensemble (PIDE) ((Friedrich et al. 2013)) indicates that for protons the $10 \%$ survival RBE grows linearly with LET, ranging from 1 $(\mathrm{LET} \simeq 1 \mathrm{keV} / \mu \mathrm{m})$ to above $2.5(\mathrm{LET} \simeq 50 \mathrm{keV} / \mu \mathrm{m})$. As primary protons slow down in tissue, their LET increases and the RBE changes accordingly. Another source of RBE variation is represented by secondary particles (also referred to as target fragments) of different species and energies yielded by nuclear interactions of the primary beam with tissue (Durante \& Paganetti 2016). In addition to providing a boost of dose to the primary protons, the charged and neutral fragments give a significant, and eventually the only, contribution to the dose off-beam, i.e. in the 
out-of-field region (Grassberger \& Paganetti 2011). Their LET can reach values well above the protons and their RBE can span from $\simeq 1$ to over 7 (Friedrich et al. 2013). Although the overall dose deposited out-of-field is lower than in-field, a high LET could lead to dramatic biological outcomes as suggested by the RBE values.

A recent workshop co-hosted by the Children's Oncology Group and the National Cancer Institute (Haas-Kogan et al. 2018) focused on the incidence of brainstem injuries following brain tumor treatments with proton therapy for pediatric patients. One of the outcomes of the discussion was that LET hotspots out-of-beam were recognized as a potential cause of different types of injuries (as radionecrosis) outside the tumor because of their increased biological effectiveness. The same concept of linking high LET regions and normal tissue toxicity in proton therapy has been explored by recent studies (Giantsoudi et al. 2016, Grosshans et al. 2018, Underwood et al. 2018), which prove the controversial nature of this issue and thus the need to address it in greater detail.

In this work, we assess cell survival RBE in- and out-of-field from measurable physical quantities characteristics of the radiation field. We use microdosimetry for this purpose. This methodology takes into account the stochastic nature of energy deposition in microscopic targets and provides a direct measurement of the lineal energy $y$ (the microdosimetric equivalent of the LET) for single particles, from which the dose can be obtained. The RBE is then evaluated using a modified version of the Microdosimetric Kinetic Model (MKM) (Hawkins 2013) developed by Kase et al. (Kase et al. 2013). Microdosimetry has been already exploited for characterizing the radiation field quality and assess the RBE in proton therapy (De Nardo et al. 2004, Kase et al. 2013, Burigo et al. 2015, Tran et al. 2017, Takada et al. 2018, Zhu et al. 2019) but so far all studies have focused on the in-field or end-of-range regions. The novelty of this work is to focus on the off-beam regions lateral to the field with a special attention on the edges, where the dose gradient is expected to be very high. The data presented here include microdosimetry lineal energy spectra as well as dose, RBE and RBE-weighted dose profiles measured inand off-beam at several depths in water. The results identify dose and LET hot spots, which can reveal potential critical regions for normal tissue toxicity during proton therapy. 


\section{Methods}

\section{Experimental setup}

The measurements presented in this paper were carried out at the experimental room of the Trento Protonterapy Center (Italy) (Tommasino et al. 2017, Tommasino et al. 2018). All microdosimetry spectra have been measured with a walled Tissue Equivalent Proportional Counter-TEPC (type LET-1/2, Far West Technology, Inc., Goleta, CA, USA). The detector sensitive volume is a sphere of $12.7 \mathrm{~mm}$ diameter, whose walls are made of A-150 tissue-equivalent plastic and are $1.27 \mathrm{~mm}$ thick. The sphere is filled with a propane proportional gas whose pressure is adjusted to achieve a density of $1.08 \cdot 10^{-3} \mathrm{~g} \mathrm{~cm}^{-3}$. Under these conditions, the detector simulates a tissue-equivalent sphere of $2 \mu \mathrm{m}$ diameter. The gas sphere is housed in an $0.18 \mathrm{~mm}$ thick Aluminum shell to maintain the desired pressure and to allow the detector to be placed in liquid targets.

The TEPC was placed inside a water phantom (model Blue phantom, IBA) and exposed to a $154 \mathrm{MeV}$ proton pencil beam. The elements between the exit window and the water phantom (including the air gaps) degraded the protons initial energy and enlarge the beam spot size. These parameters were measured at the phantom entrance using the MLIC (MultiLayer Ionization Chamber) and Lynx detectors (Tommasino et al. 2017), respectively. The results indicated that the protons residual energy was $152 \mathrm{MeV}$ (159 mm range in water) and that the FWHM of the beam spot was $13.3 \mathrm{~mm}$ on both planes perpendicular to the propagation direction. The number of primary ions impinging on the water phantom was monitored with a $3 \mathrm{~mm}$ plastic scintillator and an ion chamber $(0.5 \mathrm{~mm}$ water equivalent thickness). To avoid pile up effects, the proton rate was set to have an event rate at the TEPC around $5 \mathrm{kHz}$. A scheme of the experimental setup is shown in Fig. 1 (top panel). A similar setup had previously been used for carbon ions (Martino et al. 2010).

To select the measurements positions, the TEPC was centered on the beam axis using a laser system. Taking into account the beam width at the entry channel and the detector size, four different regions were identified as of interest for this study:

- In-beam region, where the detector was fully and homogeneously irradiated by the primary beam;

- beam-edge region, where the detector was moved $20 \mathrm{~mm}$ laterally from the beam 

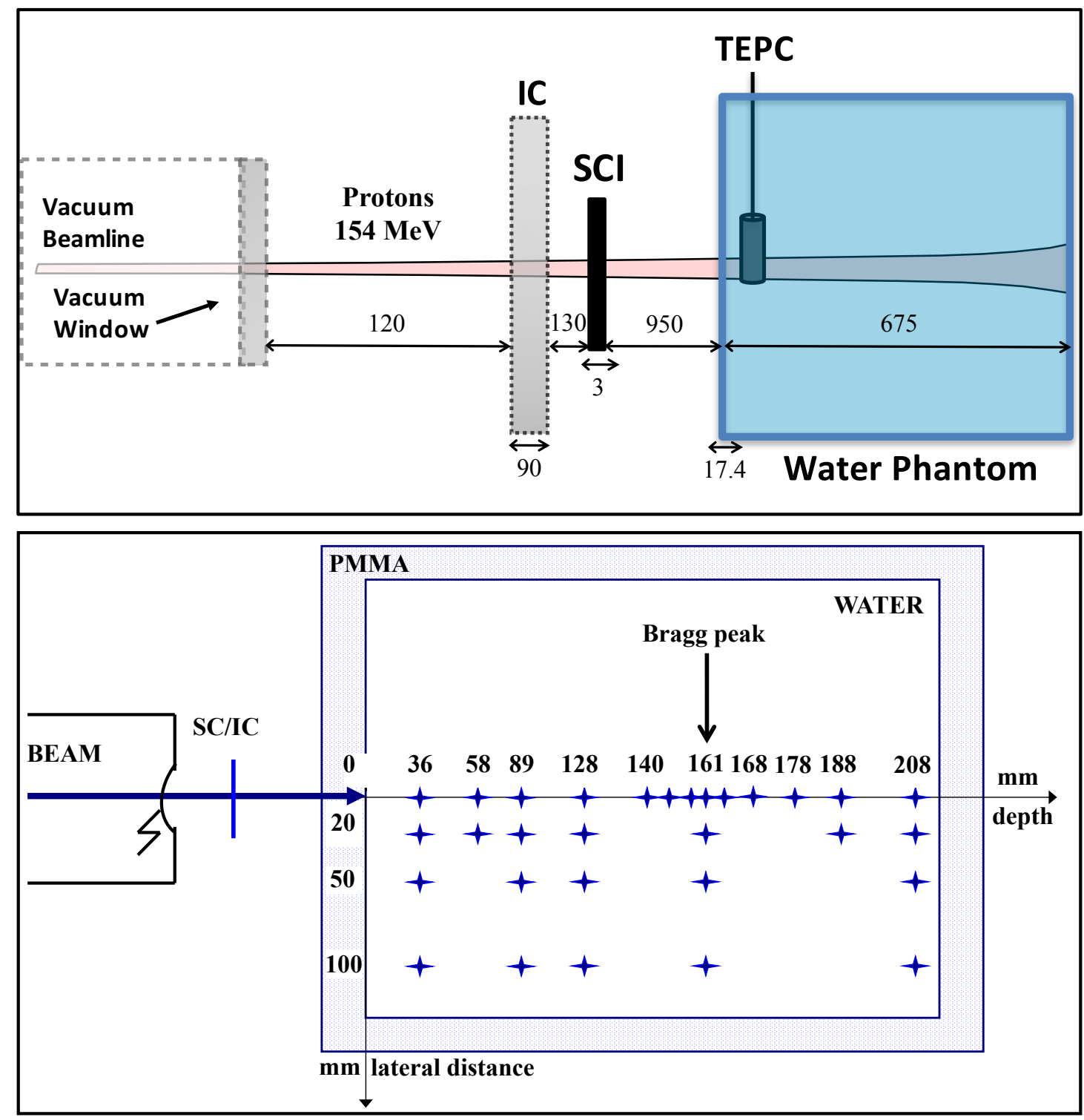

Figure 1: Scheme of the experimental setup (top panel) and of the TEPC measurement positions inside the water phantom (bottom panel). All distances and thicknesses are in $\mathrm{mm}$. The depth also takes into account the water phantom wall, whose thickness is expressed in water equivalent. The expected Bragg peak position at $159 \mathrm{~mm}$ depth is marked with an arrow. 
axis (i.e. just outside the primary irradiation field);

- close out-of-field region, where the detector was moved $50 \mathrm{~mm}$ laterally from the beam axis;

- far out-of-field region, where the detector was moved $100 \mathrm{~mm}$ laterally from the beam axis.

For each region, the microdosimetric spectra were acquired at different water depths both upstream and downstream of the Bragg peak. A scheme of the measurement positions inside the water phantom is shown in Fig. 1 (bottom panel). The depths take into account also the PMMA wall $(17.4 \mathrm{~mm}$ expressed in water equivalent).

\section{Analysis of the microdosimetry spectra}

The microdosimetry spectra are represented as lineal energy $y$ versus $y d(y)$ (with $d(y)=f(y) y$ and $f(y)$ the frequency of particles with a given $y$ ). The raw spectra have been converted into lineal energy spectra by applying a linear calibration, whose parameters were obtained exposing the TEPC to monoenergetic proton beams of different energies.

To characterize the radiation field quality, we used the following quantities:

- frequency-mean lineal energy (microdosimetric equivalent of the track-average LET)

$$
\bar{y}_{F}=\frac{\int y f(y) d y}{\int f(y) d y}
$$

- dose-mean lineal energy (microdosimetric equivalent of the dose-average LET)

$$
\bar{y}_{D}=\frac{\int y d(y) d y}{\bar{y}_{F}}
$$

- saturation-corrected dose-mean lineal energy

$$
y^{*}=\frac{y_{0}^{2} \int\left(1-\exp \left(-y^{2} / y_{0}^{2}\right)\right) f(y) d y}{\bar{y}_{F}}
$$

The quantity $y_{0}$ represents the saturation parameter to correct for the overkilling effect of high-LET radiation. Here, a value of $150 \mathrm{keV} / /$ mum has been assigned to $y_{0}$ (Kase et al. 2013). From the calibrated spectra, the total absorbed dose $D$ can be obtained using the microdosimetric version of the standard formula 
for a mixed radiation field (International Commission on Radiation Units and Measurements 1983):

$$
D=\frac{k}{d^{2}} \bar{y}_{F}
$$

where $k=0.204$ for spherical volumes, $d$ is the TEPC simulated diameter and $\bar{y}_{F}$ the frequency-mean lineal energy calculated according to equation(1).

By coupling the microdosimetry spectra and dose profiles with biological data, the RBE for cell survival can be assessed. This methodology relies on the Linear Quadratic (LQ) model and on a modified version of the MKM model (Kase et al. 2013). The first step is to calculate the survival fraction $S$ of cells at each measurement position:

$$
S=\exp \left[-\left(\alpha_{0}+\frac{\beta}{\rho \pi r_{d}^{2}} y^{*}\right) D+\beta D^{2}\right]
$$

The dose $D$ and the saturation-corrected dose-mean lineal energy $y^{*}$ can be estimated from the lineal energy spectra while $\rho$ is the density of tissue assumed to be $1 \mathrm{~g} \mathrm{~cm}^{-3}$. The cell radius $r_{d}$ as well as the $\alpha_{0}$ and $\beta$ parameters of the LQ model depend on the cell line. For this study, the Human Salivary Glands (HSG) tumor cells have been selected $\left(r_{d}=0.42 \mu \mathrm{m}, \alpha_{0}=0.13 \mathrm{~Gy}^{-1}, \beta=0.05 \mathrm{~Gy}^{-2}\right.$ and $\alpha / \beta=2.6$ Gy) (Kase et al. 2006). From the survival fraction, the RBE can be calculated as:

$$
R B E_{S}=\frac{D_{S, R}}{D_{S}}=\frac{\sqrt{\alpha_{R}^{2}-4 \beta \ln (S)}-\alpha_{R}}{2 \beta D_{S}}
$$

where $D_{S}$ and $D_{S, R}$ are the doses required by the radiation of interest and the reference radiation, respectively, to yield the same cell survival fraction $S$. Following the work of Kase et al. (Kase et al. 2006), x-rays at $200 \mathrm{keV}$ were chosen as reference radiation with an $\alpha_{R}$ value of $0.19 \mathrm{~Gy}^{-1}$ and a $\beta_{R}$ value of $0.05 \mathrm{~Gy}^{-2}$.

It is important to remark that in this study the cell survival from which the RBE is estimated is not a fixed value (e.g. 10\%), but depends on the dose measured in that position and is calculated using equation (5).

\section{Data uncertainty}

The overall error is the sum of the systematic component stemming from the lineal energy calibration of the microdosimetry spectra, the reproducibility component 
coming from the setup procedure (e.g. the detector alignment) and the statistical uncertainties.

To estimate the systematic error, we varied the calibration parameters within their error bars and applied them to the raw spectra. We then assessed how different calibrations effected the microdosimetry parameters. Using this method, we obtained an uncertainty of $8 \%$ on $\bar{y}_{F}$, of $15 \%$ on $\bar{y}_{D}$ and of $12 \%$ on $y^{*}$.

Reproducibility was assessed by acquiring the same data point in different experimental campaigns. The results yielded an error of $3 \%$ on $\bar{y}_{F}$, of $14 \%$ on $\bar{y}_{D}$ and of $5 \%$ on $y^{*}$.

The statistical uncertainties proved to be negligible compared to the other two contributors due to the large number of events collected for each measurement.

The errors on dose, RBE and dose-weigthed RBE were obtained propagating the uncertainties on $\bar{y}_{F}$ and $y^{*}$ according to the standard theory.

\section{Results}

\section{Lineal energy spectra}

The microdosimetric lineal energy spectra, $y d(y)$, in their standard semi-log representation, are presented in-beam and off-beam at several depths in water. All spectra range from 0.03 to $1000 \mathrm{keV} / \mu \mathrm{m}$ and are normalized so that the integral of the $\mathrm{yf}(\mathrm{y})$ distribution is equal to 1 .

To understand the features of the spectra it is useful to divide the radiation field into its three main components: primary protons, secondary ions and secondary neutrons. The latter two are generated by nuclear interactions of the proton beam with water and are also referred to as target fragments. A detailed description of the relevant nuclear physics concepts can be found in (Tommasino \& Durante 2015, Durante \& Paganetti 2016, Rovituso \& La Tessa 2017). The number of primary protons that undergo fragmentation and thus produce at least a secondary particle has been estimated to be around $1 \%$ per $\mathrm{cm}$ of water traversed (Tommasino \& Durante 2015). The primary beam is fairly monoenergetic at the entrance channel, and thus has a specific lineal energy, but its spectrum becomes wider when it approaches the Bragg peak region due to the energy straggling. Instead, target fragments can be neutrons and charged particles of different species and energies, producing a broad $y$ spectrum at any depth. The majority of secondary ions are 


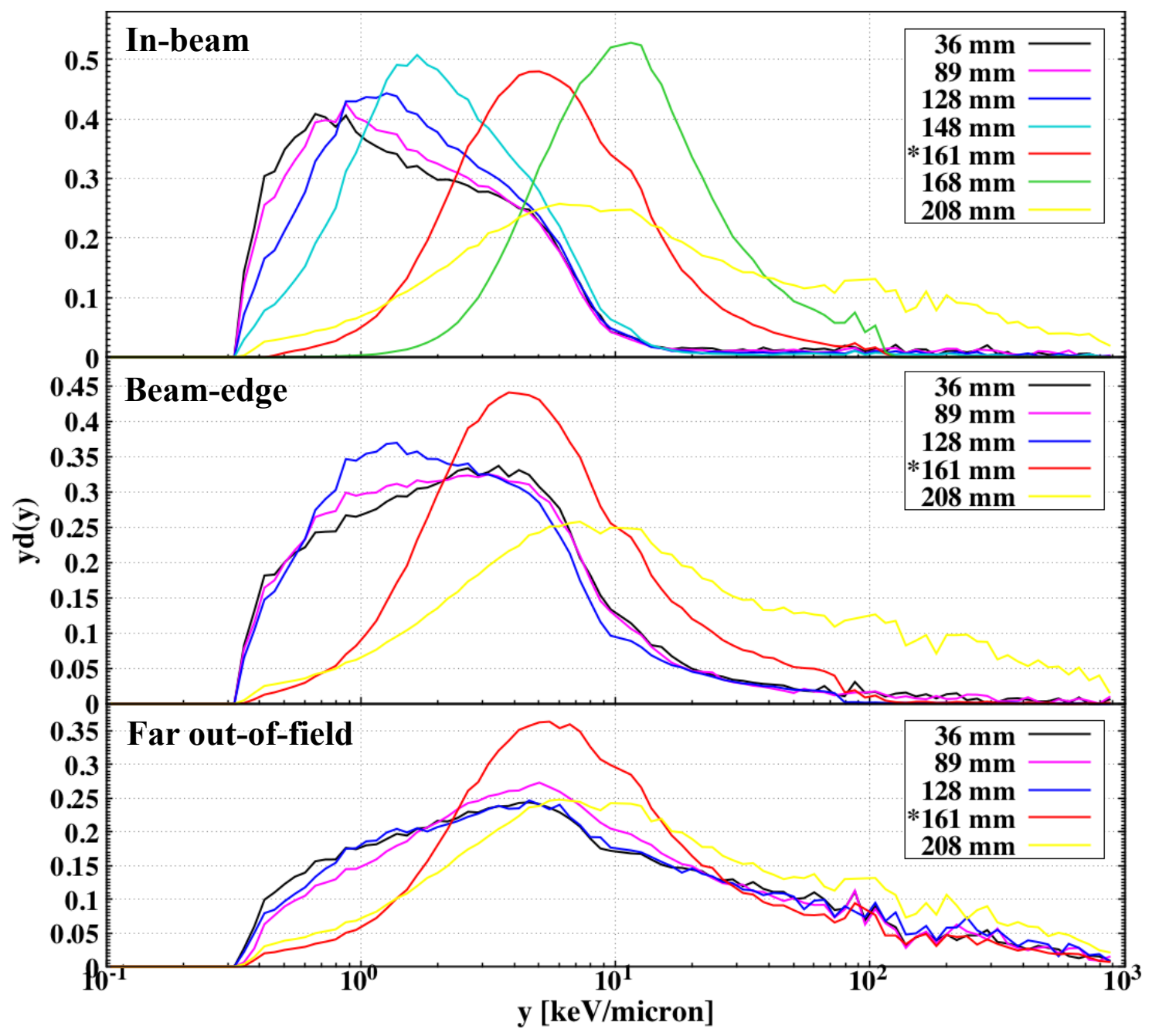

Figure 2: In- and off-beam microdosimetric lineal energy spectra $y d(y)$ measured at several depths. The off-beam regions are defined as beam-edge $(20 \mathrm{~mm}$ from the beam axis) and far out-of-field (100 $\mathrm{mm}$ from the beam axis). The asterisk indicates the spectra measured at the Bragg peak depth. All spectra are normalized so that the integral of the $\mathrm{yf}(\mathrm{y})$ distribution is equal to 1. 
generated with lower energy than the primary protons and because they have the same or higher charge, their stopping point will be upstream or around the Bragg peak depth. Because of their physical properties, neutrons are the only fragment type that do not follow this behavior and can reach sites very far from the main beam. For this reason, they become an important source (and eventually the only source) of dose both downstream of the Bragg peak and at increasing lateral distance from the beam axis. Results presented by Dewey et al. (Dewey et al. 2017) provide a summary of all these considerations, showing the contribution to the dose of each fragments species produced by a primary proton beam in water, when a $y$-based weight is applied.

All microdosimetry data presented here can be interpreted on these bases.

At the entrance channel (black curves of figure 2), the spectrum measured in-beam has a rather defined maximum around $0.7 \mathrm{keV} / \mu \mathrm{m}$ and extend up to 10 $\mathrm{keV} / \mu \mathrm{m}$. Very few events can be observed at higher lineal energy. Moving away from the beam, the distributions become flatter and broader, eventually stretching across the whole $y$ range. The low- $y$ peak corresponds to the primary beam, which can reach also the out-of-field region because of lateral scattering in water while the remaining contribution is given by the target fragments.

In-beam, the peak of primary protons moves to higher $y$ and gets wider at increasing water depth. As protons slow down their lineal energy increases and reaches its maximum in the Bragg peak region (figure 2 red curves), where their average $y$ is around $5 \mathrm{keV} / \mu \mathrm{m}$. Both the beam-edge and far out-of-field spectra measured upstream of the Bragg peak appear very similar, indicating a radiation field rather constant and independent of the depth. At the Bragg peak $(161 \mathrm{~mm})$, the distributions resemble the spectrum in-beam. This behaviour is caused by the fact that most primary and secondary protons will stop around this depth.

Independently of the lateral distance, the high- $y$ channels of all spectra become more populated at increasing depth. The reason for this trend is that the probability for the primary protons to either undergo lateral scattering or produce a target fragment increases with increasing depth. Secondary particles can be produced at large angles with respect to the primary beam direction and can also suffer lateral scattering being deflected even further from the beam axis. In addition, they can yield further generations of fragments, whose production point can be already outof-field.

In the tail region, (figure 2 yellow curves), all primary protons as well as most 
charged fragments have stopped. The spectra loose almost completely the low- $y$ region (below $1 \mathrm{keV} / \mu \mathrm{m}$ ) and extend to very high-y (above $100 \mathrm{keV} / \mu \mathrm{m}$ ).

\section{Radiation quality and dose}

The frequency-mean lineal energy $\bar{y}_{F}$ (equation (1)), dose-mean lineal energy $\bar{y}_{D}$ (equation (2)) and saturation-corrected dose-mean lineal energy $y^{*}$ (equation (3)) obtained from the $y f(y)$ and $y d(y)$ spectra, respectively, are plotted in figure 3.

Independently of the lateral position, the $\bar{y}_{F}, \bar{y}_{D}$ and $y^{*}$ appear rather flat up to the Bragg peak region and then begin to increase. The growth is more pronounced in-beam than out-of-field. The highest values are always observed downstream of the Bragg peak depth. The $\bar{y}_{F}$ curve in-beam is the only one that shows a distinct maximum while all the others seem to reach a plateau. The difference between $\bar{y}_{D}$ and $y^{*}$ is always significant with the exception of the Bragg peak region in-beam.

The microdosimetric quantities $\bar{y}_{F}, \bar{y}_{D}$ and $y^{*}$ describe the quality of the radiation field and thus they are affected if its composition changes both in terms of particle type and energy. In-beam, the field is a mix of primary and secondary radiation while at increasing lateral depth most charged particles range out and eventually only neutrons remain.

Using equation (4) the dose can be estimated from the microdosimetry spectra. To allow for a direct comparison, all measurements have been scaled to deliver a dose of 2 Gy at the Bragg peak position and thus they will be reported in Gy/2Gy-BP. The results are plotted in figure 4 and summarized in Table 1.

The dose distribution inside the water phantom has been also simulated with PHITS Monte Carlo transport code (Nose et al. 2005, Niita et al. 2006). In this case, the size of the detection volume has been chosen small enough $\left(1 \mathrm{~mm}^{3}\right.$ voxel size $)$ to obtain a 2D color map, which is presented in figure 5 .

The dose measured in-beam slowly rises from the entrance channel up to the Bragg peak. The position of the latter has been found at $161 \mathrm{~mm}$ depth, which agrees with the range of $152 \mathrm{MeV}$ protons in water measured independently with the MLIC detector. After the peak, the dose drops below $10^{-4}$ Gy/2Gy-BP within 15 $\mathrm{mm}$.

The simulated 2D plots indicate that the overall dose in-beam is dominated by primary protons (figure 5, panel a and c), as expected. The contribution from secondary particles is rather flat along the whole curve and around $0.2 \mathrm{~Gy} / 2 \mathrm{~Gy}-\mathrm{BP}$. 


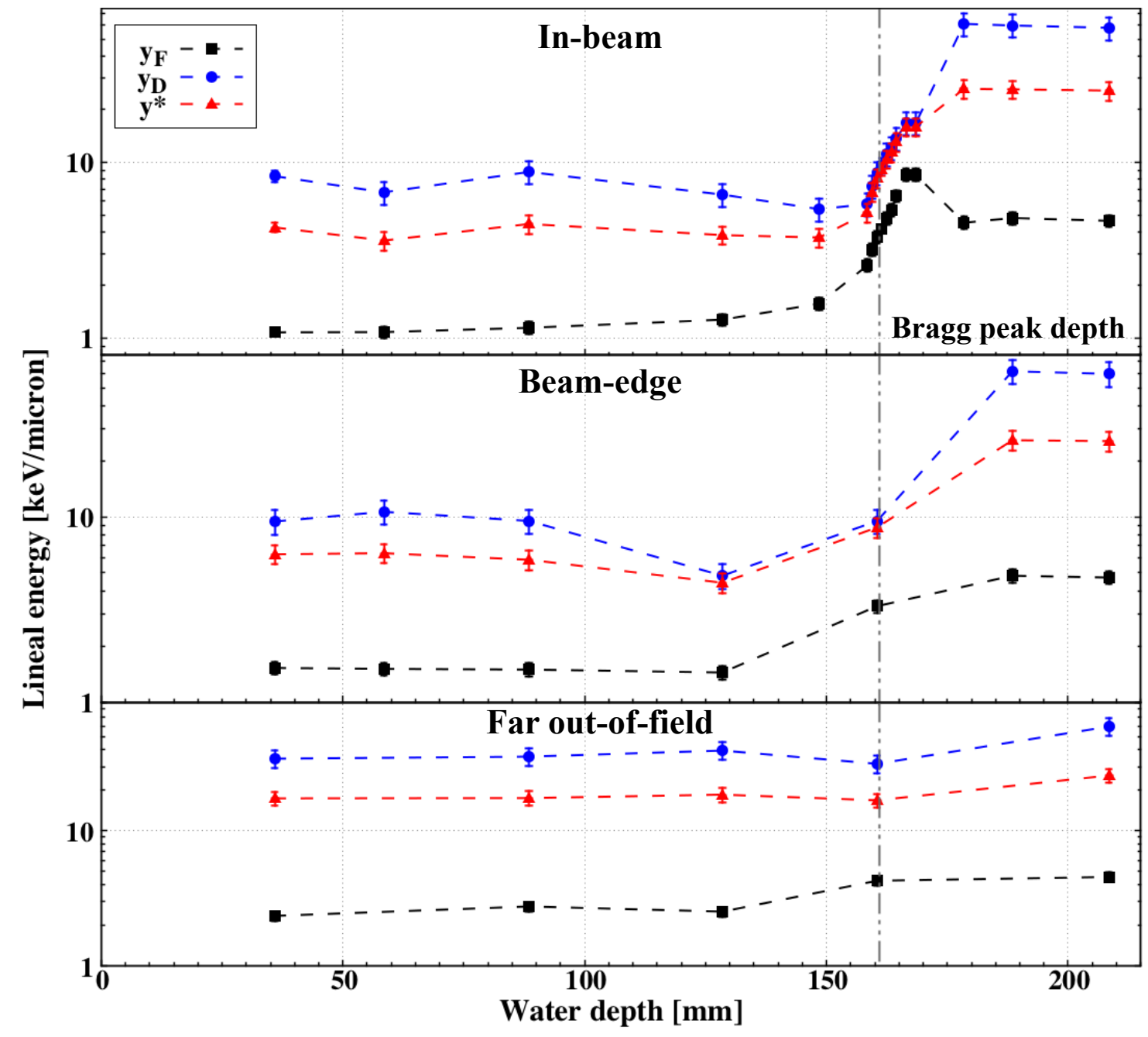

Figure 3: Frequency-mean lineal energy $\bar{y}_{F}$ (equation (1)), dose-mean lineal energy $\bar{y}_{D}$ (equation (2)) and saturation-corrected dose-mean lineal energy $y^{*}$ (equation (3)) plotted as a function of the depth in water. The off-beam regions are defined as beamedge (20 $\mathrm{mm}$ from the beam axis) and far out-of-field (100 $\mathrm{mm}$ from the beam axis). The dotted line marks the Bragg peak position (161 mm). 


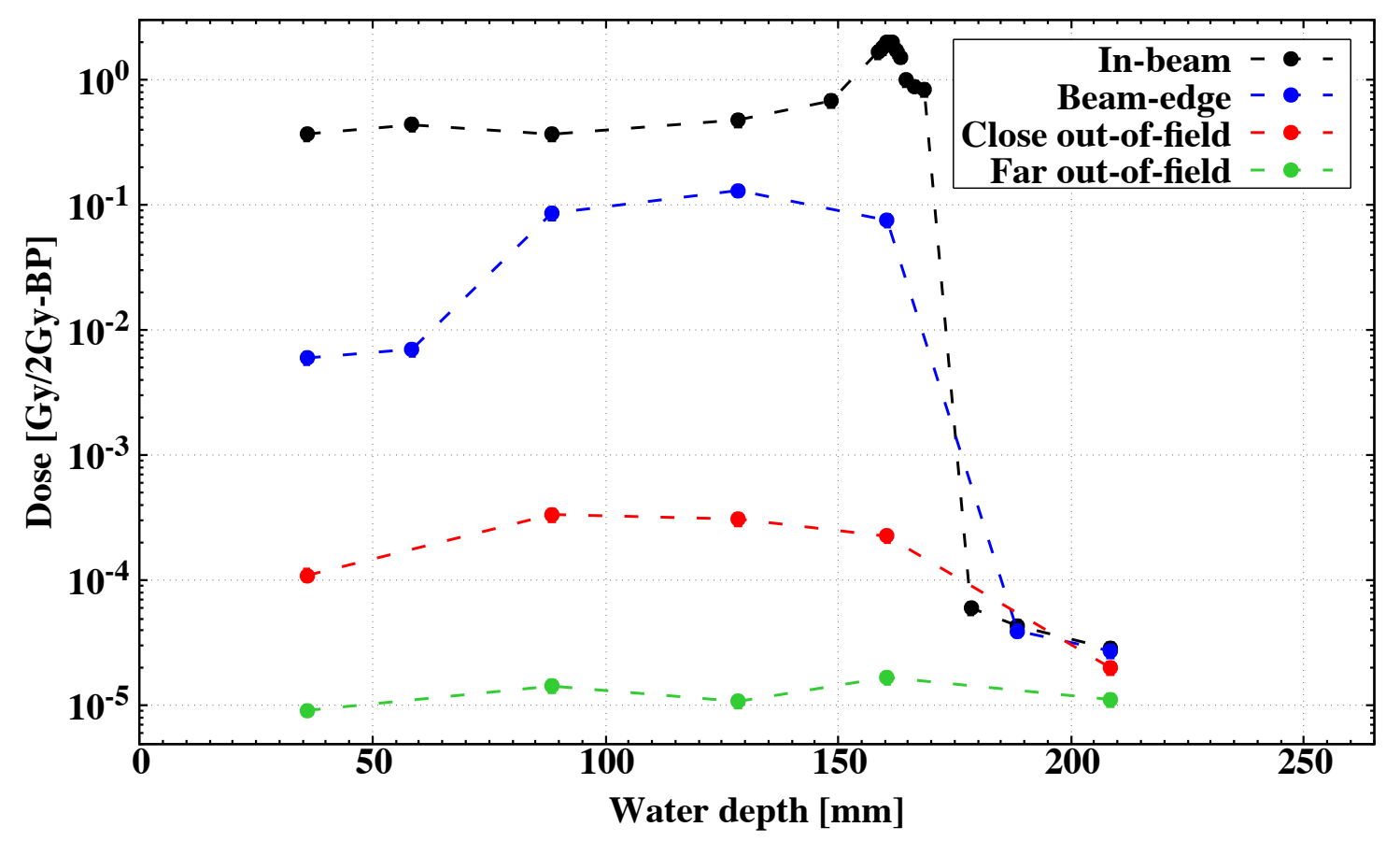

Figure 4: Dose profiles in and off beam when 2 Gy are delivered to the Bragg peak position. The off beam regions are defined as beam-edge $(20 \mathrm{~mm}$ from the beam axis), close out-of-field (50 $\mathrm{mm}$ from the beam axis) and far out-of-field (100 $\mathrm{mm}$ from the beam axis).

Then, it drops sharply several orders of magnitudes within few $\mathrm{mm}$ from the Bragg peak position, indicating that the range of most fragments do not exceed that of the primary protons.

The curve measured at the beam-edge show a rather large build-up and proves that a non negligible amount of dose is deposited in this area, reaching its maximum $\left(1.31 \pm 0.1510^{-1} \mathrm{~Gy} / 2 \mathrm{~Gy}-\mathrm{BP}\right)$ upstream of the Bragg peak depth. The simulation indicates that the overall dose in this region (panel a) is in of the order of $10^{-2}$ Gy/2Gy-BP at the entrance channel and grows up to $10^{-1} \mathrm{~Gy} / 2 \mathrm{~Gy}-\mathrm{BP}$ around the Bragg peak depth, in agreement with the experimental data. Secondary particles (panel c) deposit most of the overall dose while the contribution from primary protons scattered in this region increases with increasing depth and reaches its maximum around the Bragg peak. 
Table 1: Dose (equation (4)), RBE (equation (6)) and RBE*Dose measured inbeam and out-of-field. For each position, the lateral distance from the beam axis and the water depth are reported in parentheses. All doses are measured when $2 \mathrm{~Gy}$ are delivered to the Bragg peak and thus are reported as Gy/2Gy-BP.

\begin{tabular}{|c|c|c|c|c|}
\hline \multicolumn{2}{|c|}{$\begin{array}{l}\text { Position } \\
(\mathrm{mm})\end{array}$} & $\begin{array}{c}\text { Dose } \\
(\mathrm{Gy} / 2 \mathrm{~Gy}-\mathrm{BP})\end{array}$ & $\mathrm{RBE}$ & $\begin{array}{c}\text { RBE*}^{*} \text { Dose } \\
(\mathrm{Gy} / 2 \mathrm{~Gy}-\mathrm{BP})\end{array}$ \\
\hline \multirow{6}{*}{ In-beam (0) } & Entrance (36) & $(3.75 \pm 0.04) 10^{-1}$ & $1.01 \pm 0.09$ & $(3.72 \pm 0.04) 10^{-1}$ \\
\hline & Plateau (128) & $(4.81 \pm 0.05) 10^{-1}$ & $0.98 \pm 0.18$ & $(4.72 \pm 0.02) 10^{-1}$ \\
\hline & Rise (148) & $(6.82 \pm 0.08) 10^{-1}$ & $0.98 \pm 0.18$ & $(6.66 \pm 0.14) 10^{-1}$ \\
\hline & Bragg peak (161) & $2.0 \pm 0.2$ & $1.18 \pm 0.04$ & $2.35 \pm 0.13$ \\
\hline & Tail (178) & $(6.1 \pm 0.7) 10^{-5}$ & $2.68 \pm 0.10$ & $(1.62 \pm 0.19) 10^{-4}$ \\
\hline & Tail & $(2.9 \pm 0.3) 10^{-5}$ & $2.63 \pm 0.10$ & $(7.5 \pm 0.9) 10^{-5}$ \\
\hline \multirow{4}{*}{ Beam-edge (20) } & Entranc & $(6.0 \pm 0.7) 10^{-3}$ & $1.16 \pm 0.10$ & $(7.0 \pm 1.0) 10^{-3}$ \\
\hline & Rise(1 & $(1.31 \pm 0.15) 10^{-1}$ & $1.02 \pm 0.18$ & $(1.3 \pm 0.3) 10^{-1}$ \\
\hline & Bragg pea & $(7.6 \pm 0.9) 10^{-3}$ & $1.34 \pm 0.11$ & $(1.01 \pm 0.14) 10^{-1}$ \\
\hline & Tail (208) & $(2.7 \pm 0.3) 10^{-5}$ & $2.63 \pm 0.10$ & $(7.2 \pm 0.9) 10^{-5}$ \\
\hline \multirow{4}{*}{ Close-out (50) } & Entrance (36) & $(1.10 \pm 0.12) 10^{-4}$ & $1.45 \pm 0.08$ & $(1.6 \pm 0.2) 10^{-4}$ \\
\hline & Rise(128) & $(3.1 \pm 0.4) 10^{-4}$ & $1.33 \pm 0.15$ & $(4.1 \pm 0.7) 10^{-4}$ \\
\hline & Bragg peak (161) & $(2.3 \pm 0.3) 10^{-4}$ & $1.57 \pm 0.11$ & $(3.6 \pm 0.5) 10^{-4}$ \\
\hline & Tail (208) & $(2.0 \pm 0.3) 10^{-5}$ & $2.60 \pm 0.06$ & $(5.2 \pm 0.6) 10^{-5}$ \\
\hline \multirow{4}{*}{ Far-out (100) } & Entrance (36) & $(9.1 \pm 1.0) 10^{-6}$ & $2.01 \pm 0.06$ & $(1.8 \pm 0.2) 10^{-5}$ \\
\hline & Rise(128) & $(1.08 \pm 0.12) 10^{-5}$ & $2.10 \pm 0.11$ & $(2.3 \pm 0.3) 10^{-5}$ \\
\hline & Bragg peak (161) & $(1.69 \pm 0.19) 10^{-5}$ & $1.97 \pm 0.13$ & $(3.3 \pm 0.4) 10^{-5}$ \\
\hline & Tail (208) & $(1.12 \pm 0.13) 10^{-5}$ & $2.65 \pm 0.07$ & $(3.0 \pm 0.3) 10^{-5}$ \\
\hline
\end{tabular}

At larger lateral distances, the experimental curves are rather flat and present a small drop just downstream of the Bragg peak. The maximum dose in the closeout-of-field region is one order of magnitude higher than in the far-out-of-field area. The Monte Carlo predictions (figure 5, panel b) indicate that the dose is around $10^{-5} \mathrm{~Gy} / 2 \mathrm{~Gy}-\mathrm{BP}$ at $50 \mathrm{~mm}$ off-beam and decreases to $10^{-6} \mathrm{~Gy} / 2 \mathrm{~Gy}-\mathrm{BP}$ at $100 \mathrm{~mm}$ off-beam. Panel d of figure 5 suggests that secondary particles becomes the only contributors to the dose in these areas. 




Figure 5: 2D color plots of the dose deposition inside the water phantom simulated with PHITS Monte Carlo code (Nose et al. 2005, Niita et al. 2006) when 2 Gy are delivered to the Bragg peak position. The contribution from primary protons and secondary fragments is shown in panel (a) inside the entire water phantom and panel (b) as a zoom around the beam axis. The contribution only from secondaries is displayed in panel (c) inside the entire water phantom and panel (d) as a zoom around the beam axis. In all plots, the off-beam regions defined as beam-edge (20 $\mathrm{mm}$ from the beam axis), close out-of-field (50 $\mathrm{mm}$ from the beam axis) and far out-of-field (100 $\mathrm{mm}$ from the beam axis) are marked with dashed lines.

It is interesting to notice the dose measured at $250 \mathrm{~mm}$ depth drops to $10^{-5}$ Gy/2Gy-BP independently of the region, indicating a uniform radiation field. At the same depth, the simulated overall dose is one order of magnitude lower (i.e. $10^{-6}$ Gy/2Gy-BP) and it is entirely delivered by the fragments.

In summary, the measured and simulated dose profiles suggest that there is a non negligible number of primary ions that deviate enough from their initial path to deposits some dose out-of-field. However, as the distance from the beam-edge increases, the secondary particles become the major and eventually the only source 
of dose. Assuming that the total dose delivered to the tumor in a full treatment is $60 \mathrm{~Gy}$, the normal tissue will receive up to 4 Gy at the field edge, $10^{-2}$ Gy in the close-out-of-field region and $5^{*} 10^{-4}$ Gy in the far-out-of-field region.

\section{$R B E$ and $R B E$-weighted dose}

The microdosimetry spectra and dose profiles have been combined to obtain an estimate of the RBE for cell death, according to equations 5 and 6 . It is important to underline that especially out-of-field, the RBE is measured for a mixed radiation field composed of both the primary protons and target fragments. The results are reported in Table 1 and plotted in figure 6 (top panel). A full line at 1.1 marks the reference value for protons while a dotted line indicates the Bragg peak depth (161 $\mathrm{mm})$.

All datasets show the same trend, with an extended plateau followed by a rise near the Bragg peak depth. In-beam, all RBE values measured upstream of the peak agree within the uncertainties with the reference value of 1.1. The RBE found at the Bragg peak is $1.18 \pm 0.05$ and increases up to $2.68 \pm 0.10$ at a depth of $178 \mathrm{~mm}$, which is also the overall highest value observed. The curve measured at the field edge follows the data in-beam, showing very similar values. Out-of-field, the RBE are always higher than 1.1 and increases with increasing lateral distance. Downstream of the Bragg peak all regions are characterized by a rather constant and similar RBE around 2.6.

The rather large RBE variation are due to the target fragments, whose lineal energy can be substantially higher than that of primary protons as demonstrated by the $y d(y)$ spectra (figure 2). In the entrance channel, the RBE is always rather constant, indicating that most particles have a similar $y$. In-beam and at the field edge, most particles are either primary protons or fast fragments with low $y$. The out-of-field region, instead, is mostly populated by slower secondary particles, whose $y$ is higher, and thus the RBE is well above 1.1. At increasing depth, both primary ions and fragments slow down, their lineal energy increases and so does the RBE.

Although the RBE represents an indicator of the radiation effectiveness, it has to be combined with the dose to assess the biological outcomes. Thus, the data from figure 5 (top panel) have been multiplied with the corresponding dose measurements (figure 4) to obtain the RBE-weighted dose curves plotted in figure 5(bottom panel). The high RBE values observed outside the target area are in most cases heavily 


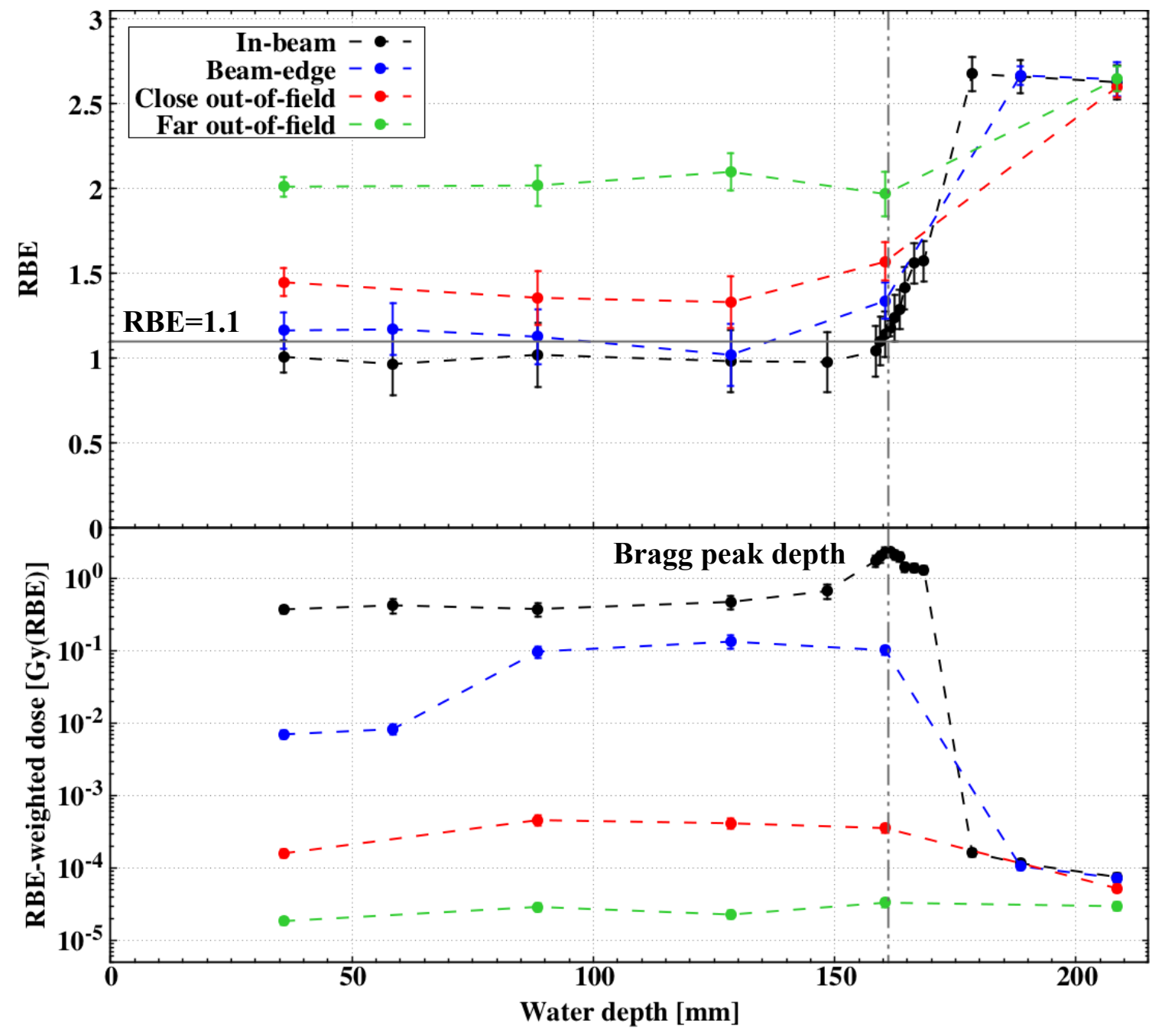

Figure 6: Top panel: In- and off-beam RBE values as a function of water depth. The off-beam regions are defined as beam-edge (20 $\mathrm{mm}$ from the beam axis), close out-of-field (50 $\mathrm{mm}$ from the beam axis) and far out-of-field (100 $\mathrm{mm}$ from the beam axis). All RBE values have been calculated according to equation (6) when 2 Gy are delivered to the Bragg peak. The reference protons value of 1.1 is marked with a full line, while the Bragg peak depth $(161 \mathrm{~mm})$ is marked with a dotted line. Bottom panel: RBE-weighted dose in- and off-beam as a function of water depth. The offbeam regions are defined as beam-edge (20 $\mathrm{mm}$ from the beam axis), close out-of-field (50 $\mathrm{mm}$ from the beam axis) and far out-of-field (100 $\mathrm{mm}$ from the beam axis). The results have been obtained multiplying the dose (Fig. 4) with the corresponding RBE value for each position. 
moderated by the dose, which drops below $10^{-3}$ Gy out-of-field. Together with the end-of-range region in-beam, these data point to the field-edge as a potential area at risk of toxicity.

\section{Discussion}

Finding a solid bridge between the physical parameters describing a radiation field and its corresponding biological effects, and translating them into clinically relevant results represents one of the most difficult challenges in radiotherapy. To address this issue, the National Cancer Institute (NCI) created a dedicated panel, whose task was to identify the most suitable set of physics factors for characterizing a particle beam (Durante et al. 2019). When applied to clinical research, these recommendations would improve the intercomparison of different biological experiments and ultimately facilitate the understanding of the relation between physics parameters related to the treatment and patients outcomes. The report produced by the panel included also a list of standard measurements recommended for obtaining a detailed characterization of the beam. Microdosimetry was listed among the techniques for investigating the beam purity and the quality of the radiation field.

Along the same lines, the absorbed dose alone does not carry enough information for assessing the biological and, ultimately, the clinical effects of particle beams. A full characterization of the radiation field in terms of particle species and kinetic energy (or LET) as well as information on the radiation track structure are necessary tools to predict the biological damage (Conte et al. 2017). Aside from providing a better understanding of the particle effectiveness in tumor killing, these data can also help predicting the risk of undesired effects following the treatment. In fact, the concept that normal tissue toxicity occurring either at the target edges or out-offield might be caused to high-LET particles has been recently discussed (Haas-Kogan et al. 2018).

In this work, a methodology for characterizing the beam quality, including the assessment of the RBE for cell survival from the combination of physical and biological quantities has been developed. The approach is based on two types of inputs: microdosimetry data, which provide lineal energy $y$ spectra as well as absorbed dose, and cell survival curves predicted with the LQ and MKM models. The study presented here focused on the characterization of a $152 \mathrm{MeV}$ therapeutic 
proton beam and included both measurements in- and off-beam at several water depths. The microdosimetry spectra (figure 2) indicate that the number of high lineal energy particles $(y \geq 10 \mathrm{keV} / \mu \mathrm{m})$ increases with increasing depth both in- and -out-of-field. This trend translates into a rapid RBE growth (figure 4), which reaches a maximum value of $\approx 2.6$ downstream of the Bragg peak and in the lateral regions. The dose profiles indicate that for a full treatment of 60 Gy delivered to the tumor, the overall dose received by the normal tissue is as high as 4 Gy at the field-edge, $10^{-2} \mathrm{~Gy}$ in the close-out-of-field region and $5^{*} 10^{-4} \mathrm{~Gy}$ in the far-out-of-field region.

Microdosimetry has been already exploited to obtain the radiation field quality in protontherapy. The measurements were performed with different types of detectors including spherical TEPCs (Kase et al. 2013) similar to that used in this work, wall-less TEPCs (Tsuda et al. 2012), miniaturized cylindrical TEPCs (De Nardo et al. 2004) and silicon on insulator (SOI) microsensors (A Wroe 2007, Wroe et al. 2009, Tran et al. 2017). TEPCs are currently considered the gold standard for microdosimetry because of their equivalence to biological tissue and sensitivity to low$y$ particles. However, commercial models have a limited spatial resolution due to their size (over $10 \mathrm{~mm}$ diameter for the sensitive volume) and would suffer heavy pile up effects if exposed to therapeutic particle rates. Miniaturized TEPCs were developed to partially solved these issues while maintaining the tissue-equivalence (Colautti et al. 2018). Semiconductor microsensors (Rosenfeld 2016), on the other hand, can reach submillimeter spatial resolutions and sustain high fluxes, but their detection threshold for low- $y$ particles is more limited than the TEPCs and their results rely on the silicon-to-tissue-equivalent conversion.

Additional microdosimetry studies have been carried out using Monte Carlo codes (Burigo et al. 2015, Dewey et al. 2017, Mairani et al. 2017, Takada et al. 2018, Zhu et al. 2019), with the main purpose of validating the models against experimental data and to use the MKM model for RBE assessment. A further step to bridge microdosimetry and clinical outcomes has been done by Ardenfors et al. (Ardenfors et al. 2018), who combined microdosimetric measurements and Monte Carlo calculations to estimate the risk of radiation-induced second cancer in a brain tumor treatment with protons.

An overview of the microdosimetry results and RBE values presented here as well as of the experimental and simulated data available in literature is presented in Table 2. We identified three regions of interest for the comparison: plateau and Bragg peak depths in-field, tail in the out-of-field region downstream of the peak. 
Table 2: Comparison of microdosimetry results presented in this work with data available in literature. The plateau and peak regions are in-beam while the tail is the out-of-field area proximal to the Bragg peak. References marked with * contain data taken with a Spread Out Bragg Peak (SOBP) while ** indicate Monte Carlo

simulations. Values reported without uncertainties have been estimated from plots.

\begin{tabular}{|c|c|c|c|c|c|}
\hline Region & $\begin{array}{l}\text { Reference } \\
\mathrm{keV} / m u m\end{array}$ & $\begin{array}{c}\bar{y}_{F} \\
\mathrm{keV} / m u \mathrm{~m}\end{array}$ & $\begin{array}{c}\bar{y}_{D} \\
\mathrm{keV} / m u \mathrm{~m}\end{array}$ & $y^{*}$ & RBE \\
\hline \multirow{6}{*}{ Plateau } & This work & $1.27 \pm 0.10$ & $6.5 \pm 1.0$ & $3.6 \pm 0.5$ & $0.98 \pm 0.18$ \\
\hline & (De Nardo et al. 2004)* & - & - & - & 1.1 \\
\hline & (Kase et al. 2013) & 0.8 & 5 & 4 & $0.95 \pm 0.02$ \\
\hline & (Tran et al. 2017) & - & 2 & - & $0.96 \pm 0.14$ \\
\hline & (Takada et al. 2018)** & - & - & - & 0.98 \\
\hline & (Dewey et al. 2017) $)^{* *}$ & - & - & 4 & 0.98 \\
\hline \multirow{6}{*}{ Peak } & This work & $4.2 \pm 0.2$ & $9.6 \pm 1.0$ & $9.1 \pm 0.8$ & $1.18 \pm 0.05$ \\
\hline & (De Nardo et al. 2004)* & - & - & - & 1.2 \\
\hline & (Kase et al. 2013) & 6 & 18 & 8 & 1.15 \\
\hline & (Tran et al. 2017) & - & 7 & - & $1.10 \pm 0.08$ \\
\hline & $($ Takada et al. 2018)** & - & - & - & 1.1 \\
\hline & (Dewey et al. 2017) $)^{* *}$ & - & - & 3 & 1.0 \\
\hline \multirow{6}{*}{ Tail } & This work & $8.5 \pm 0.7$ & $16 \pm 2$ & $15.9 \pm 1.9$ & $1.58 \pm 0.12$ \\
\hline & (De Nardo et al. 2004)* & - & - & - & 2.3 \\
\hline & (Kase et al. 2013) & 7 & - & 17 & 1.8 \\
\hline & (Tran et al. 2017) & - & - & - & $1.57 \pm 0.19$ \\
\hline & (Takada et al. 2018)** & - & 10 & - & 1.8 \\
\hline & (Dewey et al. 2017) ${ }^{* *}$ & - & - & 13 & 1.7 \\
\hline
\end{tabular}

The reason for choosing these areas is because we consider them as representative of the radiation field. Furthermore, with the exception of the tail region, we could not compare any out-of-field microdosimetry data because they are not included in any of the previous studies.

416 The agreement between the studies varies significantly depending on the considered quantity, with the largest discrepancy observed for $\bar{y}_{D}$. The latter is 
the most sensitive to high- $y$ events, whose frequency is rather rare and thus is very affected by statistics as already discussed in (Kase et al. 2006). All other quantities are in a fair agreement considering the difference of experimental setups, detector types and beam energies.

In summary, the present work as well as the published experimental and Monte Carlo studies on microdosimetry indicated that coupling physical quantities with biological models can provide a reasonable estimate of the RBE for cell survivals. If in principle this approach is ideal, as it could be applied to any clinical case (e.g. tumor type or particle species), its main limitation is that the accuracy heavily depends on both the physical and biological models implemented in the code. Experimental data that provide information as detailed as possible on the radiation field composition as well as measured survival curves for several cell lines are necessary to improve this promising methodology.

\section{Conclusions}

In this paper, an approach for assessing the RBE for cells survivals by combining microdosimetry and biological data as well as LQ and MKM predictions has been presented. This approach has been applied to a clinical monoenergetic proton beam delivered in a water phantom to obtain data both in- and out-of-field. These results can help revealing the link between dose, lineal energy and toxicity effects, especially in the beam-edge region, where the dose reach values as high as 4 Gy for a $60 \mathrm{~Gy}$ treatment. LET- and RBE-based optimizations of treatment planning in proton therapy have been proposed (Paganetti 2014, Haas-Kogan et al. 2018) to mitigate the risk of toxicity especially at the protons end-of-range and at the field edges. The data presented here represent a fundamental base for developing this strategy.

\section{Acknowledgments}

The authors thank the microdosimetry group of INFN-LNL (Italy) and in particular Valeria Conte for the many precious suggestions and useful discussions that helped improving the experimental setup as well as gaining a better insight of the data. 


\section{References}

A Wroe, A Rosenfeld R S 2007 Out-of-field dose equivalents delivered by proton therapy of prostate cancer Med. Phys. 34(9), 3349-3456.

Ardenfors O, Dasu A, Lillhök J, Persson L \& Gudowska I 2018 Out-of-field doses from secondary radiation produced in proton therapy and the associated risk of radiation-induced cancer from a brain tumor treatment Phys. Med. 53, 129-136.

Burigo L, Pshenichnov I, Mishustin I \& Bleicher M 2015 Comparative study of dose distributions and cell survival fractions for ${ }^{1} \mathrm{H},{ }^{4} \mathrm{He},{ }^{12} \mathrm{C}$ and ${ }^{16} \mathrm{O}$ beams using Geant4 and Microdosimetric Kinetic model Phys. Med. Bio. 60, 3313-3331.

Colautti P, Conte V, Selva A, Chiriotti S, Pola A, Bortot D, Fazzi A, Agosteo S, Treccani M, De Nardo L, Verona C, Rinati G, Magrin G, Cirrone G \& Romano F 2018 Miniaturized microdosimeters as LET monitors: First comparison of calculated and experimental data performed at the $62 \mathrm{MeV} / \mathrm{u}^{12} \mathrm{C}$ beam of INFN-LNS with four different detectors Phys. Med. 52, 113-121.

Conte V, Selva A, Colautti P, Hilgers G \& Rabus H 2017 Track structure characterization and its link to radiobiology Radiat. Meas. 106, 506-511.

De Nardo L, Cesari V, Iborra N, Conte V, Colautti P, Hérault J, Tornielli G \& Chauvel P 2004 Microdosimetric assessment of Nice therapeutic proton beam biological quality Phys. Med. 20, 71-77.

Dewey S, Burigo L, Pshenichnov I, Mishustin I \& Bleicher M 2017 Lateral variations of radiobiological properties of therapeutic fields of ${ }^{1} \mathrm{H},{ }^{4} \mathrm{He},{ }^{12} \mathrm{C}$ and ${ }^{16} \mathrm{O}$ ions studiede with Geant4 and microdosimetric kinetic model Phys. Med. Bio. 62, 5884-5907.

Durante M \& Loeffler J S 2010 Charged particles in radiation oncology Nature Rev. Clin. Oncol. 7, 37-43.

Durante M \& Paganetti H 2016 Nuclear physics in particle therapy: a review Rep. Prog. Phys. 79, 096702.

Durante M, Paganetti H, Pompos A, Kry S, Wu X \& Grosshans D 2019 Report of a National Cancer Institute special panel: Characterization of the physical parameters of particle beams for biological research Med. Phys. In press.

Friedrich T, Scholz U, Durante M \& Scholz M 2014 RBE of ion beams in hypofractionated radiotherapy (SBRT) Med. Phys. 30, 588-591.

Friedrich T, Scholz U, Elsässer T, Durante M \& Scholz M 2013 Systematic analysis of RBE and related quantities using a database of cell survival experiments with ion beam irradiation $J$. Radiat. Res. 54, 494-514.

Giantsoudi D, Sethi R V, Yeap B Y, Eaton B R, Ebb D H, Caruso P A, Rapalino O, Chen Y L E, Adams J A, Yock T I, Tarbell N J, Paganetti H \& MacDonald S M 2016 Incidence of CNS injury for a cohort of 111 patients treated with proton therapy for medulloblastoma: LET and RBE associations for areas of injury Int. J. Radiat. Oncol. Biol. Phys. 95, 287-296.

Grassberger C \& Paganetti H 2011 Elevanted LET components in clinical proton beams Phys. Med. Bio. 56, 6677-6691.

Grosshans D R, Duman J G, Gaber M W \& Sawakuchi G 2018 Particle radiation induced 
neurotoxicity in the central nervous system IJPT 5, 74-83.

Haas-Kogan D, Indelicato D, Paganetti H, Esiashvili N, Mahajan A, Yock T, Flampouri S, MacDonald S, Fouladi M, Stephen K, Kalapurakal J, Terezakis S, Kooy H, Grosshans D, Makrigiorgos M, Mishra K, Poussaint T, Cohen K, Fitzgerald T, Gondi V, Liu A, Michalski J, Mirkovic D, Mohan R, Perkins S, Wong K, Vikram B, Buchsbaum J \& Kun L 2018 National cancer institute workshop on proton therapy for children: Considerations regarding brainstem injury Int. J. Radiat. Oncol. Biol. Phys. 101, 152-168.

Hawkins R 2013 A microdosimetric-kinetic theory of the dependence of the RBE for cell death on LET Med. Phys. 40, 0011705.

International Commission on Radiation Units and Measurements 1983 Microdosimetry ICRU 36.

Kase Y, Kanai T, Matsumoto Y, Furusawa Y, Okamoto H, Asaba T, Sakama M \& Shinoda H 2006 Microdosimetric measurements and estimation of human cell survival for heavy-ion beams Rad, Res. 166, 629-638.

Kase Y, Yamashita W, Matsufuji N, Takada K, Sakae T, Furusawa Y, Yamashita H \& Murayama S 2013 Microdosimetric calculation of relative biological effectiveness for design of therapeutic proton beams J. Radiat. Res. 54, 485-493.

Mairani A, Magro G, Tessonnier T, Böhlen T, Molinelli S, Ferrari A, Parodi K, Debuss J \& Haberer T 2017 Optimizing the modified microdosimetric kinetic model input parameters for proton and ${ }^{4}$ he ion beam therapy application Phys. Med. Bio. 62, 244-256.

Martino G, Schardt D \& Durante M 2010 Microdosimetry measurements characterizing the radiation field of $300 \mathrm{MeV} / \mathrm{u}{ }^{12} \mathrm{C}$ and $185 \mathrm{MeV} / \mathrm{u}{ }^{7} \mathrm{Li}$ pencil beams stopping in water Phys. Med. Bio. 55, 3441-3449.

Niita K, Sato T, Iwase H, Nose H, Nakashima H \& Sihver L 2006 PHITS - a particle and heavy ion transport code system Radiat. Meas. 41, 1080.

Nose H, Niita K, Hara M, Uematsu K, Azuma O, Miyauchi Y, Komori M \& Kanai T 2005 Improvement of three-dimensional Monte Carlo code PHITS for heavy ion theraphy $\mathrm{J}$. Nucl. Sci. Tech. 42, 250.

Paganetti H 2014 Relative biological effectiveness (RBE) values for proton beam therapy. variations as a function of bioogical endpoint, dose, and linearl energy transfer Phys. Med. Bio. 59, R419-R472.

Rosenfeld A 2016 Novel detectors for silicon based microdosimetry, their concepts and applications NIMA 809, 156-170.

Rovituso M \& La Tessa C 2017 Nuclear interactions of new ions in cancer therapy: impact on dosimetry Transl. Cancer Reas. 6, 1310-1326.

Takada K, Sato T, Kumada H, Koketsu J, Takei H, Sakurai H \& Takeji S 2018 Validation of the physical and rbe-weighted dose estimator based on PHITS coupled with a microdosimetric kinetic model for proton therapy J. Radiat. Res. 59, 91-99.

Tommasino F \& Durante M 2015 Proton radiobiology Cancers 7, 353-381.

Tommasino F, Rovituso M, Bortoli E, La Tessa C, Petringa G, Lorentini S, Verroi E, Simenov Y, Weber U, Cirrone P, Schwarz M, Durante M \& Scifoni E 2018 A new facility for proton radiobiology at the Trento Proton Therapy centre: design and implementationy Phys. Med. 58, 99-106. 
Tommasino F, Rovituso M, Fabiano S, Piffer S, Manea C, Lorentini S, Lanzone S, Wang Z, Pasini M, Burger W J, La Tessa C, Scifoni E, Schwarz M \& Durante M 2017 Proton beam characterization in the experimental room of the Trento Proton Therapy facility NIMA $\mathbf{8 6 9}, 15-20$.

Tran L T, Chartier L, Bolst D, Pogossov A, Guatelli S, Petasecca M, Lerch M L F, Prokopovich D A, Reinhard M I, Clasie B, Depauw N, Kooy H, Flanz J B, McNamara A, Paganetti H, Beltran C, Furutani K, Perevertaylo V L, Jackson M \& Rosenfeld A B 2017 Characterization of proton pencil beam scanning and passive beam using a high spatial resolution solid-state microdosimeter Med. Phys. 44(11), 6085-6095.

Tsuda S, Sato T, Takahashi F, Satoh D, Sasaki S, abd H. Iwase Y N, Ban S \& Takada M 2012 Systematic measurement of lineal energy distributions for proton, He and $\mathrm{Si}$ ion beams over a wide energy range using a wall-less tissue equivalent proportional counter $J$. Radiat. Res. 53, 264-271.

Underwood T S, Grassberger C, Bass R, MacDonald S M, Meyersohn N M, Yeap B Y, Jimenez R B \& Paganetti H 2018 Asymptomatic late-phase radiographic changes among chest-wall patients are associated with a proton rbe exceeding 1.1 Int. J. Radiat. Oncol. Biol. Phys. 101, $809-819$.

Wroe A, Clasie B, Kooy H, Flanz J, Schulte R \& Rosenfeld A 2009 Out-of-field dose equivalents delivered by passively scattered therapeutic proton beams for clinically relevant field configurations Int. J. Radiat. Oncol. Biol. Phys. 73(1), 306-313.

Zhu H, Chen Y, Sung W, McNamara A L, Tran L T, Burigo L N, Rosenfeld A B, Li J, Faddegon B, Schuemann J \& Paganetti H 2019 The microdosimetric extension in TOPAS: development and comparison with published data Phys. Med. Bio. 64, 145004-145019. 